\title{
Chronic stress, visceral obesity and gonadal dysfunction
}

\author{
Ioannis Kyrou, ${ }^{1,2}$ Constantine Tsigos ${ }^{1}$
}

${ }^{1}$ Endocrinology, Metabolism and Diabetes Unit, Evgenidion Hospital, Athens University Medical School, Athens, Greece, ${ }^{2}$ Unit for Diabetes and Metabolism, WISDEM, University Hospitals Coventry and Warwickshire, Warwick Medical School, University of Warwick, Coventry, UK

\begin{abstract}
Chronic stress represents a prolonged state of dyshomeostasis caused by intense and frequently imposed stressors. Obesity constitutes a chronic dysmetabolic state, leading progressively to a spectrum of metabolic complications, such as diabetes, dyslipidemia, hypertension and cardiovascular disease. A growing body of evidence supports the existence of significant interactions between stress and obesity, with chronic stress promoting weight gain, and consequently excessive fat accumulation especially visceral, all these factors contributing to the development of a chronic stressful state. Maintaining body homeostasis is a prerequisite for normal reproductive function, which is vital for the survival of the species and an important process of natural selection. Under chronic stress, reproductive function is suspended and disrupted due to central and peripheral actions of hormones, adipokines and pro-inflammatory cytokines that inhibit the activity of the hypothalamic-pituitary gonadal (HPG) axis at various levels. Clinical and experimental data link both obesity and chronic stress to dysregulation of the gonadal axis, via independent and synergistic mechanisms, which may chronically lead to reproductive dysfunction and reduced fertility.
\end{abstract}

Key words: Abdominal obesity, Adipokines, Gonads, Hypogonadism, Hypothalamic-pituitaryadrenal axis, Obesity, Sex-steroids, Stress

\section{INTRODUCTION}

During the course of evolution most of the human physiologic systems have adapted, through strenuous processes of natural selection, to optimally serve two primary objectives, namely, survival of the self and survival of the species. Thus, complex, interwoven pathways have developed in the central nervous

Address for correspondence:

Constantine Tsigos, 82 Vas. Sophias Avenue, GR-11528

Athens, Greece, Tel: +30 210 7480009, Fax: +30 2107480010 , e-mail: ctsigos@gmail.com

Received 01-08-08, Revised 10-09-08, Accepted 20-09-08 system (CNS) and the periphery, aiming primarily at securing energy and metabolic homeostasis, and secondarily at facilitating reproduction.

The modern way of life in industrialized societies is characterized by increased intake of processed food, sedentary lifestyle and increased psychological stress, forming an environment that differs completely from that of our predecessors, which has driven human evolution until recently. Thus, it is not surprising that the rates of obesity and stress-related complications have been exponentially on the increase over the past decades. Recent data indicate a strong interplay 
between stress and obesity, which also directly affects the activity of the hypothalamic-pituitary gonadal (HPG) axis. This article briefly reviews potential pathophysiologic mechanisms which link chronic stress and visceral obesity and may lead to metabolic complications and reproductive dysfunction.

\section{STRESS SYSTEM AND THE PHYSIOLOGY OF THE STRESS RESPONSE}

Stress can be defined as a state of threatened homeostasis and is counteracted by an intricate repertoire of physiologic and behavioral responses that collectively aim to re-establish the disturbed equilibrium (adaptive stress response) ${ }^{1,2}$ The adaptive stress response is facilitated by an elaborate neuro-endocrine, cellular and molecular infrastructure, the stress system, which extends both throughout the CNS and the periphery. The central components of the stress system include: 1) the parvocellular corticotropin-releasing hormone $(\mathrm{CRH})$ and arginine-vasopressin (AVP) neurons of the hypothalamic paraventricular nuclei (PVN) and the $\mathrm{CRH}$ neurons of the paragigantocellular and parabranchial nuclei of the medulla and 2) the locus ceruleus/norepinephrine system (LC/NE) and other catecholaminergic cell groups of the medulla and pons (central sympathetic system). The peripheral limbs of the hypothalamic-pituitary-adrenal (HPA) axis and the efferent sympathetic/adrenomedullary system constitute the primary peripheral components of this complex homeostatic system (Figure 1).

The adaptive stress response is determined by genetic, environmental and developmental factors, which dictate the characteristics of the mobilized defenses against intrinsic and extrinsic stressors. Inappropriate responses to stressors, as for example inadequate, excessive and/or prolonged reactions, may turn deleterious and contribute to disease. Furthermore, chronically imposed or severe stressors can impair various physiologic functions, such as metabolism, reproduction, immunity, growth, as well as personality development and behavior. ${ }^{3}$ As part of the adaptive stress response, non-vital, energy-demanding functions (e.g. reproductive and digestive functions) are transiently inhibited in order to preserve energy and to promote a redirection of oxygen and nutrients to the CNS and stressed body site(s), where they are needed the most. Thus, chronic

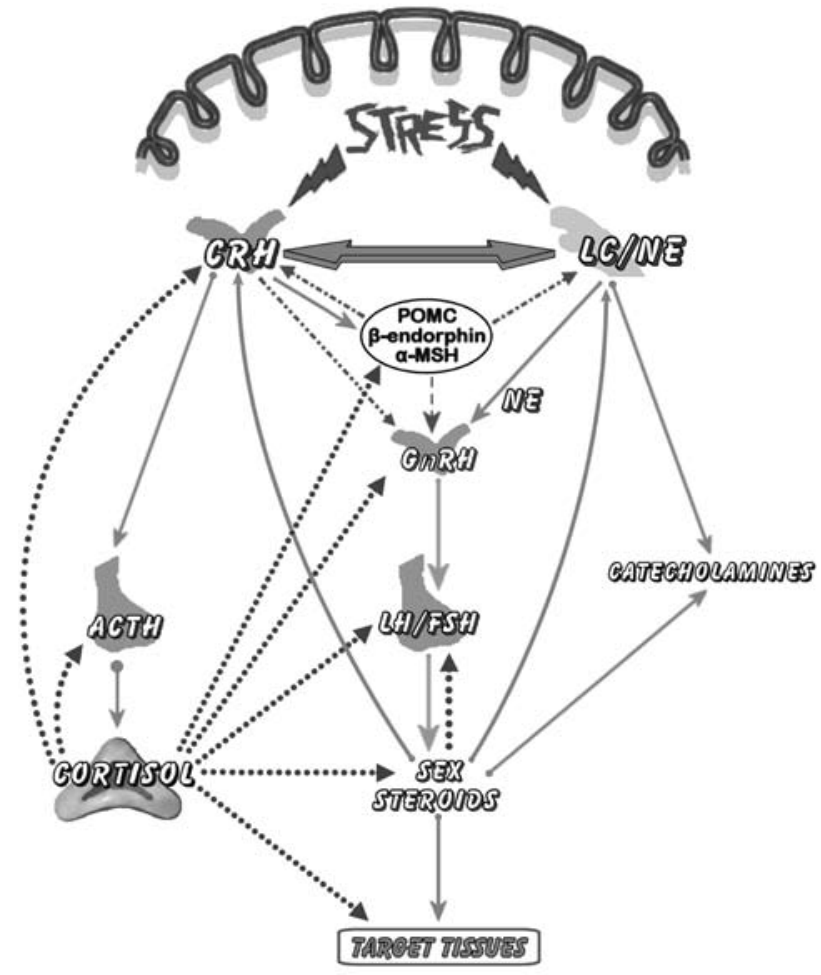

Figure 1. Interactions between the stress system and the hypothalamic-pituitary-gonadal axis. LC/NE: locus ceruleus/norepinephrine-sympathetic system, CRH: corticotropin-releasing hormone, POMC: proopiomelanocortin, $\alpha$-MSH: $\alpha$-melanocyte-stimulating hormone, GnRH: gonadotropin-releasing hormone, ACTH: adrenocorticotropic hormone, LH: luteinizing hormone, FSH: follicle-stimulating hormone, NE: norepinephrine. Stimulatory effects are represented by solid lines and inhibitory effects by dashed lines.

stress, which can be defined as a pathological state of prolonged threat to homeostasis by persistent or frequently repeated stressors, may progressively lead to reproductive dysfunction and reduced fertility. ${ }^{4}$

\section{INTERPLAY BETWEEN THE HYPOTHALAMIC- PITUITARY - ADRENAL AND HYPOTHALAMIC- PITUITARY GONADAL AXES}

HPA axis activation exerts multiple inhibitory actions on the HPG axis (Figure 1). The gonadotropinreleasing hormone (GnRH) neurons of the arcuate nucleus are suppressed by $\mathrm{CRH}$, both directly and indirectly, via enhanced $\beta$-endorphin secretion from arcuate POMC neurons. In addition, glucocorticoids have inhibitory effects on the GnRH neurons, the pituitary gonadotroph cells (primarily on the secre- 
tion of LH) and the gonads (where they directly inhibit steroidogenesis). Furthermore, glucocorticoids render target tissues of sex-steroids resistant to these hormones. ${ }^{5,6}$ It is of note that cytokines (e.g. interleukin-6, tumor necrosis factor- $\alpha$ ), which are secreted in states of inflammatory stress and have been shown to stimulate the HPA axis, also suppress the reproductive axis at several levels by inhibiting the pulsatile hypothalamic secretion of GnRH and the ovarian or testicular steroidogenesis. ${ }^{7}$

$\mathrm{CRH}$ and HPG axis interactions appear to be bidirectional. Estrogen response elements have been identified in the promoter area of the CRH gene and direct stimulatory estrogen effects on $\mathrm{CRH}$ gene expression have also been shown. ${ }^{8}$ Thus, the CRH gene, and consequently the HPA axis, is an important target of ovarian steroids and a potential mediator of gender-related differences in HPA axis activity and stress responses. ${ }^{9}$ The female reproductive system provides positive input to both components of the stress system through estradiol, which stimulates $\mathrm{CRH}$ secretion and inhibits re-uptake and catabolism of catecholamines (Figure 1). ${ }^{10}$

Chronic stress leads to prolonged suppression of gonadal function, primarily via chronic activation of the HPA axis. This effect has been well demonstrated in ballet dancers, highly trained runners and athletes of both sexes. ${ }^{11,12}$ These individuals can present diminished ACTH responses to exogenous $\mathrm{CRH}$ administration, increased 24-hour urinary-free cortisol excretion and elevated circulating concentrations of cortisol and ACTH in the evening. Furthermore, in male subjects low LH and testosterone concentrations are detected, while females usually develop menstrual disorders and amenorrhea.

\section{CHRONIC STRESS, VISCERAL OBESITY AND METABOLIC COMPLICATIONS}

Glucocorticoids, the final hormonal effectors of the HPA axis, exert a wide range of effects on metabolism, which are primarily catabolic in an effort to utilize every available energy resource against the challenge enforced by stressors. Chronic stress prolongs this adaptive shift of metabolism towards a generalized catabolic state and, thus, sustained HPA hyperactivity can progressively lead to decreased lean body (muscle and bone) mass, increased visceral adiposity and insulin resistance through the actions of glucocorticoids on various metabolic pathways (Figure 2$).{ }^{13}$ This chronic stress-related state, characterized by the combination of decreased lean body mass, abdominal and trunk fat accumulation and manifestations of the metabolic syndrome, resembles that of Cushing's syndrome. Such cushingoid phenotypes can be identified in a variety of pathophysiologic conditions, collectively described as pseudo-Cushing's states and attributable to stress-induced mild hypercortisolism, probably in association with increased peripheral tissue sensitivity

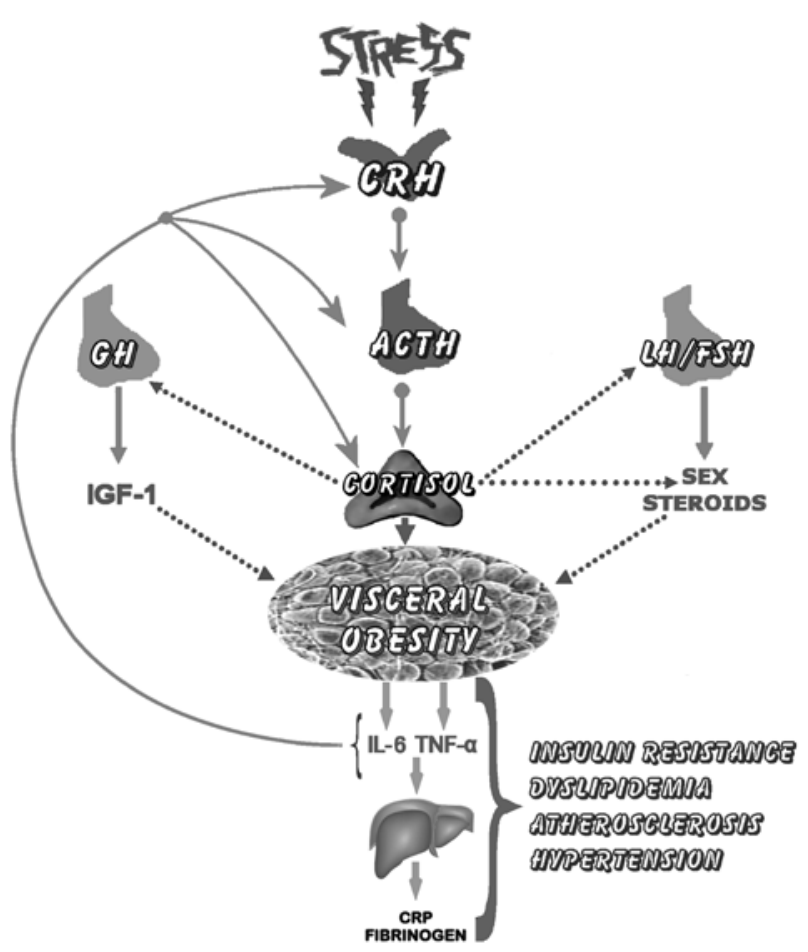

Figure 2. Reciprocal interactions between chronic stress and visceral obesity, which can lead to clinical manifestations of the metabolic syndrome. Chronic HPA axis activation favors visceral obesity and lean body (muscle and bone) mass reduction, with cortisol suppressing both the gonadal and the growth hormone axis and directly stimulating adipocyte proliferation. Reciprocally, IL- 6 and TNF- $\alpha$, overproduced by the expanding adipose tissue, further stimulate the HPA axis thus forming a vicious cycle. $\mathrm{CRH}$ : corticotropin-releasing hormone, ACTH: adrenocorticotropic hormone, LH: luteinizing hormone, FSH: follicle-stimulating hormone, $\mathrm{GH}$ : growth hormone, IGF-1: insulin-like growth factor- 1 , TNF- $\alpha$ : tumor necrosis factor- $\alpha$, IL6: interleukin-6, CRP: C-reactive protein. Stimulatory effects are represented by solid lines and inhibitory effects by dashed lines. 
to glucocorticoids. ${ }^{14,15}$ The association between chronic stressors and increased incidence of visceral obesity and metabolic syndrome is strongly supported by recent epidemiologic data, which indicate that chronic stress, including socioeconomic stress, is an important novel risk factor for the metabolic syndrome in our modern societies. ${ }^{16,17}$

Central components of the stress system are closely linked to CNS centers that control appetite and energy expenditure (Figure 3)..$^{13,18}$ Acute stress is usually associated with anorexia and consequent restriction of food consumption. $\mathrm{CRH}$ has been shown to acutely stimulate POMC neurons of the arcuate nucleus which elicit anorexic signals, via $\alpha-\mathrm{MSH}$ release, and to increase thermogenesis. Stress-induced suppression of neuropeptide Y (NPY) secretion, which has potent orexigenic and anxiolytic actions, is probably involved in causing anorexia under acute stress. However, the subsequent increase of circulating glucocorticoid concentration eventually promote the intake of carbohydrates and fat and decrease energy expenditure by suppressing CRH and stimulating NPY hypothalamic secretion. ${ }^{19,20}$ Thus, it is plausible that acute stress activates the HPA axis, through $\mathrm{CRH}$, aiming initially to inhibit temporarily the non vital, energyconsuming activities related to food consumption, whereas sustained activation of the HPA axis, under conditions of chronic stress, promotes the relatively more prolonged central actions of glucocorticoids on CNS appetite centers, which are collectively orexigenic and stress-relieving. Notably, glucocorticoid-induced orexigenic stimuli under chronic stress favor the intake of especially palatable food which is centrally perceived as a stress-relieving factor and contributes to visceral obesity. ${ }^{21-23}$

Furthermore, the adipose tissue, previously viewed as a passive energy storage depot, is now proven to function as a highly active endocrine organ, secreting multiple hormones, cytokines and other active factors. ${ }^{24}$ Adipocytes directly signal their metabolic status to organs and tissues of the periphery and to the CNS, via endocrine, paracrine, autocrine, juxtacrine and intracrine functions, forming a homeostatic circuit which is pivotal in the regulation of the energy intake and expenditure equilibrium. ${ }^{25}$ It has also become evident that centralization of body fat stores and subsequent development of visceral obes-

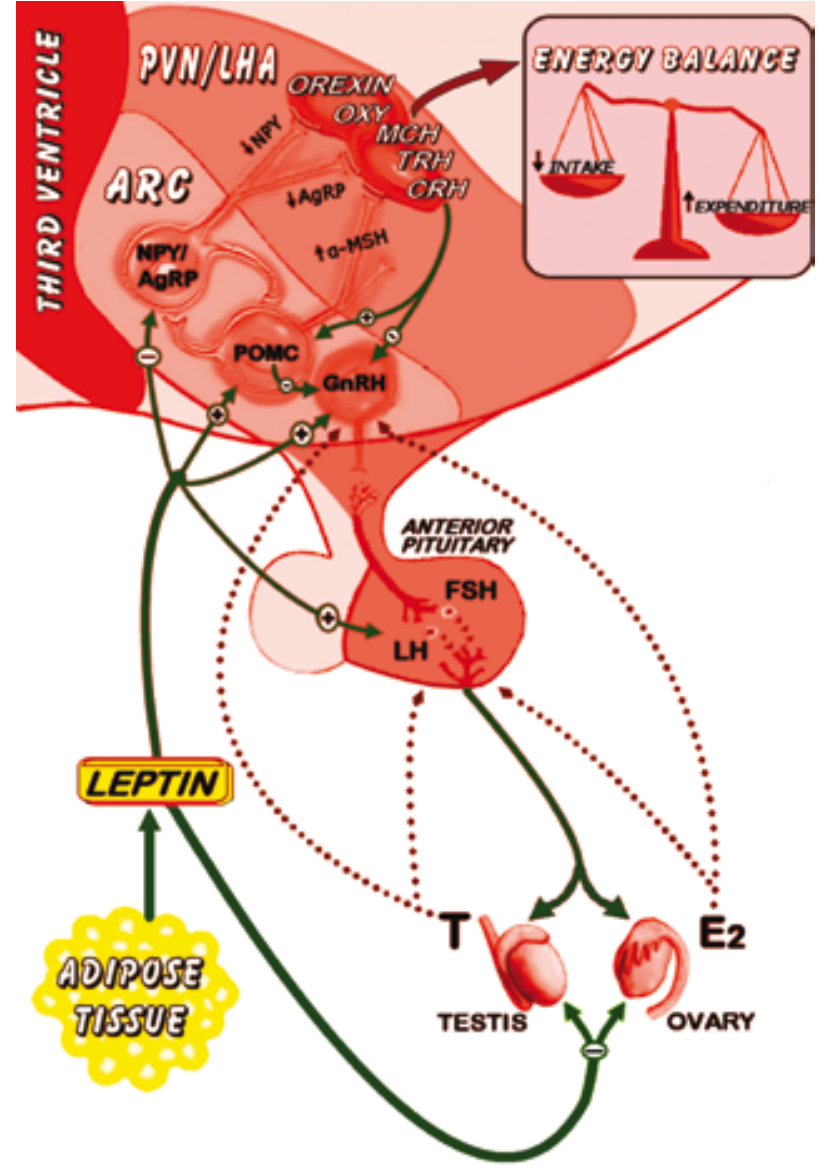

Figure 3. Interactions between adipose tissue, hypothalamic appetite-satiety centers and the hypothalamic-pituitary-gonadal axis. ARC: arcuate nucleus, PVN: paraventricular nucleus, LHA: lateral hypothalamic area, CRH: corticotropin-releasing hormone, ACTH: adrenocorticotropic hormone, POMC: proopiomelanocortin, NPY: neuropeptide Y, AgRP: agouti related peptide, $\alpha$-MSH: $\alpha$-melanocyte-stimulating hormone, Y1: neuropeptide $\mathrm{Y}$ receptor type 1, MC4R: melanocortin receptor type 4, TRH: thyrotropin-releasing hormone, $\mathrm{MCH}$ : melanin concentrating hormone, OXY: oxytocin, T: Testosterone, $\mathrm{E}_{2}$ : estradiol, LH: luteinizing hormone, FSH: follicle-stimulating hormone, GnRH: gonadotropin-releasing hormone, NE: norepinephrine. Stimulatory effects are represented by solid lines and inhibitory by dashed lines.

ity (also referred to as central, abdominal, android, upper body or apple-shaped obesity) exhibits strong associations with metabolic derangements and correlates to higher comorbidity risks. ${ }^{26,27}$ Visceral obesity is indeed considered to be the cornerstone of the metabolic syndrome pathophysiology, highlighting even more the role of adipose tissue in the pathogenesis of cardiometabolic complications, such as insulin resistance, dyslipidemia, atherosclerosis, hypertension 
and hypercoagulation. ${ }^{28}$

\section{OBESITY, CHRONIC STRESS AND GONADAL FUNCTION}

Body weight is an independent regulator of the hypothalamic-pituitary-gonadal (HPG) axis activity. This fact has been a common observation, documented in various cultures throughout history, especially in respect to the weight of women and their competence to bear children. Our understanding of the underlying physiologic mechanisms has only recently begun to evolve following the discovery of adipose tissue derived hormones (e.g. leptin, adiponectin) which have been shown to directly influence reproduction and fertility. ${ }^{29-31}$ Leptin, which primarily acts to suppress appetite and enhance energy expenditure at the hypothalamic level, appears to play a vital role in the relationship between adipose tissue and the HPG axis (Figure 3), regulating the onset of puberty, modulating reproductive capacity and facilitating implantation and pregnancy. ${ }^{32-36}$ Furthermore, the ability of adipose tissue to accumulate sex-steroids inside adipocytes and also to metabolize and interconvert them, through the actions of local enzymes, can significantly affect the functional status of the reproductive axis. ${ }^{37-40}$ Accordingly, the expanding adipose tissue mass in obesity can alter the circulating sex-steroids and the balance between bio-available estrogens and androgens. Altered plasma levels of sexsteroids and sex hormone binding globulin (SHBG) could lead to clinical manifestations of impaired HPG activity. Obesity in females has been associated with early initiation of puberty, menstrual disturbances, infertility and higher risk for certain types of breast and endometrial cancer. Increased body mass index (BMI) in obese males is correlated with higher incidence of infertility and decreased libido. ${ }^{41-47}$ Table 1 summarizes potential hormonal changes and clinical manifestations of impaired HPG axis activity in obese females and males.

Obesity also constitutes an unconventional, unremitting and low-grade inflammatory state. ${ }^{48,49}$ The increase of adipose tissue causes enhanced secretion of pro-inflammatory hormones and cytokines into the circulation (adipokines), originating from adipocytes and from macrophages that are recruited and infiltrate the expanding adipose tissue. These pro-inflammatory adipokines activate the acute phase reaction and progressively impose a generalized chronic inflammatory stress on the body. ${ }^{50,51}$ Importantly, two of the main pro-inflammatory cytokines, TNF- $\alpha$ and IL-6, are secreted in significant quantities by the enlarging adipose tissue, especially in visceral obesity. In addition to their immuno-modulating effects, TNF- $\alpha$ interferes with intracellular insulin signalling and is implicated in the pathogenesis of insulin resistance, ${ }^{52}$ while IL-6 has atherogenic effects and directly acts on other major endocrine axes (e.g. central stimulation of cortisol secretion, suppression of TSH and testosterone secretion) and on glucose and lipid metabolism. ${ }^{7,53}$ Furthermore, increased secretion of leptin (pro-inflammatory properties) and decreased

Table 1. Hormonal changes and clinical manifestations of hypothalamic pituitary gonadal (HPG) axis activity in obese males and females.

\begin{tabular}{|c|c|c|c|}
\hline \multicolumn{2}{|c|}{ HPG axis in obese females } & \multicolumn{2}{|c|}{ HPG axis in obese males } \\
\hline Hormonal changes & Clinical manifestations & Hormonal changes & Clinical manifestations \\
\hline$\leftrightarrow / \uparrow$ Estrogen & Precocious puberty - Premature menopause & $\uparrow$ Estrogen & Reduced libido \\
\hline$\uparrow$ Testosterone & Menstrual disorders & $\downarrow$ Testosterone & Erectile dysfunction \\
\hline$\downarrow$ SHBG & Chronic oligo-anovulation & $\downarrow$ SHBG & Impaired fertility \\
\hline$\leftrightarrow$ basal LH & $\begin{array}{l}\text { Impaired fertility - Poor response to fertility } \\
\text { treatment }\end{array}$ & $\leftrightarrow / \downarrow$ basal LH & \\
\hline$\leftrightarrow$ basal FSH & Increased risk of miscarriage & $\leftrightarrow / \downarrow$ basal FSH & \\
\hline$\leftrightarrow$ LH after stimulation & $\begin{array}{l}\text { Increased risk of complications during } \\
\text { pregnancy }\end{array}$ & $\leftrightarrow$ LH after stimulation & \\
\hline$\leftrightarrow$ FSH after stimulation & Increased risk of gynecologic cancer & $\leftrightarrow$ FSH after stimulation & \\
\hline
\end{tabular}

$\leftrightarrow$ : normal levels, $\downarrow$ : decreased levels, $\uparrow:$ increased levels 
secretion of adiponectin (anti-inflammatory properties) further enhance the imposed inflammatory load in obesity. This continuous supply of pro-inflammatory adipokines to the systemic circulation may act as a chronic stimulus for HPA axis activation. Indeed, interesting novel data have emerged concerning the interplay between adipocytes, HPA axis activity and cytokines, indicating that a vicious cycle may develop, whereby visceral obesity causes hypersecretion of TNF- $\alpha$ and IL- 6 that further stimulate the HPA axis, which in turn favors visceral fat accumulation and dysfunction of the HPG axis (Figure 2). Thus, hypercortisolemia appears to contribute to adipocyte accumulation and vice versa, resulting in reciprocal interactions that potentially link chronic stress to obesity and conversely obesity to chronic activation of the stress system. ${ }^{13}$

\section{CONCLUSIONS}

Reproduction is a vital process for the survival of the species and an important mechanism of natural selection. Normal reproductive function requires conditions ensuring the undisrupted activity of the HPG axis, which depends crucially on the preservation of body homeostasis. Chronic stress represents a state of dyshomeostasis (allostasis) resulting from the inability of the adaptive stress response to cope with the intensity or the frequency of various stressors. Obesity itself is a chronic, dysmetabolic state due to an unbalanced equilibrium between energy intake and expenditure and can progressively lead to an array of complications, such as diabetes and cardiovascular disease. Furthermore, prolonged stress seems to contribute to the development of obesity, especially visceral, all of which contribute to the development of a chronic stressful state. Under such unfavorable conditions, reproductive function is suspended and eventually impaired due to central and peripheral actions of hormones, adipokines and pro-inflammatory cytokines which may inhibit the activity of the HPG axis at various levels. Identification of the underlying mechanisms that mediate these actions and result in derangements of the HPG axis will provide novel insights into the reciprocal interactions between stress, obesity and reproduction thus facilitating the quest for effective therapeutic interventions.

\section{REFERENCES}

1. Chrousos GP, 2007 Organization and Integration of the Endocrine System. Sleep Med Clin 2: 125-145.

2. Chrousos GP, Gold PW, 1992 The concepts of stress and stress system disorders. Overview of physical and behavioral homeostasis. JAMA 267: 1244-1252.

3. Tsigos C, Chrousos GP, 2002 Hypothalamic-pituitary-adrenal axis, neuroendocrine factors and stress. J Psychosom Res 53: 865-871.

4. Charmandari E, Tsigos C, Chrousos G, 2005 Endocrinology of the stress response. Annu Rev Physiol 67: 259-284.

5. Rabin DS, Schmidt PJ, Campbell G, Gold PW, Jensvold M, Rubinow DR, et al, 1990 Hypothalamic-pituitary-adrenal function in patients with the premenstrual syndrome. $\mathrm{J}$ Clin Endocrinol Metab 71: 1158-1162.

6. Rivier C, Rivier J, Vale W, 1986 Stress-induced inhibition of reproductive functions: role of endogenous corticotropin-releasing factor. Science 231: 607-609.

7. Tsigos C, Papanicolaou DA, Kyrou I, Raptis SA, Chrousos GP, 1999 Dose-dependent effects of recombinant human interleukin- 6 on the pituitary-testicular axis. J Interferon Cytokine Res 19: 1271-1276.

8. Vamvakopoulos NC, Chrousos GP, 1993 Evidence of direct estrogenic regulation of human corticotropin-releasing hormone gene expression. Potential implications for the sexual dimophism of the stress response and immune/inflammatory reaction. J Clin Invest 92: 18961902.

9. Vamvakopoulos NC, Chrousos GP, 1994 Hormonal regulation of human corticotropin-releasing hormone gene expression: implications for the stress response and immune/inflammatory reaction. Endocr Rev 15: 409-420.

10. Chrousos GP, Torpy DJ, Gold PW, 1998 Interactions between the hypothalamic-pituitary-adrenal axis and the female reproductive system: clinical implications. Ann Intern Med 129: 229-240.

11. MacConnie SE, Barkan A, Lampman RM, Schork MA, Beitins IZ, 1986 Decreased hypothalamic gonadotropinreleasing hormone secretion in male marathon runners. N Engl J Med 315: 411-417.

12. Luger A, Deuster PA, Kyle SB, et al, 1987 Acute hypothalamic-pituitary-adrenal responses to the stress of treadmill exercise. Physiologic adaptations to physical training. N Engl J Med 316: 1309-1315.

13. Kyrou I, Chrousos GP, Tsigos C, 2006 Stress, visceral obesity, and metabolic complications. Ann N Y Acad Sci 1083: 77-110.

14. Chrousos GP, 2000 The role of stress and the hypothalamic-pituitary-adrenal axis in the pathogenesis of the metabolic syndrome: neuro-endocrine and target tissuerelated causes. Int J Obes Relat Metab Disord 24: Suppl 2: 50-55.

15. Kyrou I, Tsigos C, 2007 Stress mechanisms and metabolic complications. Horm Metab Res 39: 430-438.

16. Chandola T, Brunner E, Marmot M, 2006 Chronic stress 
at work and the metabolic syndrome: prospective study. BMJ 332: 521-525.

17. Brunner EJ, Chandola T, Marmot MG, 2007 Prospective effect of job strain on general and central obesity in the Whitehall II Study. Am J Epidemiol 165: 828-837.

18. Kalra SP, Dube MG, Pu S, Xu B, Horvath TL, Kalra PS, 1999 Interacting appetite-regulating pathways in the hypothalamic regulation of body weight. Endocr Rev 20: 68-100.

19. Cavagnini F, Croci M, Putignano P, Petroni ML, Invitti C, 2000 Glucocorticoids and neuroendocrine function. Int J Obes Relat Metab Disord 24: Suppl 2: 77-79.

20. Kaye WH, Berrettini W, Gwirtsman H, George DT, 1990 Altered cerebrospinal fluid neuropeptide $\mathrm{Y}$ and peptide YY immunoreactivity in anorexia and bulimia nervosa. Arch Gen Psychiatry 47: 548-556.

21. Dallman MF, Pecoraro NC, La Fleur SE, et al, 2006 Glucocorticoids, chronic stress, and obesity. Prog Brain Res 153: 75-105.

22. Dallman MF, Pecoraro N, Akana SF, et al, 2003 Chronic stress and obesity: a new view of "comfort food". Proc Natl Acad Sci USA 100: 11696-11701.

23. Torres SJ, Nowson CA, 2007 Relationship between stress, eating behavior, and obesity. Nutrition 23: 887-894.

24. Trujillo ME, Scherer PE, 2006 Adipose tissue-derived factors: impact on health and disease. Endocr Rev 27: 762-778.

25. Scherer PE, 2006 Adipose tissue: from lipid storage compartment to endocrine organ. Diabetes 55: 1537-1545.

26. Snijder MB, Zimmet PZ, Visser M, Dekker JM, Seidell JC, Shaw JE, 2004 Independent and opposite associations of waist and hip circumferences with diabetes, hypertension and dyslipidemia: the AusDiab Study. Int J Obes Relat Metab Disord 28: 402-409.

27. Lee CM, Huxley RR, Wildman RP, Woodward M, 2008 Indices of abdominal obesity are better discriminators of cardiovascular risk factors than BMI: a meta-analysis. J Clin Epidemiol 61: 646-653.

28. Alberti KG, Zimmet P, Shaw J, 2005 IDF Epidemiology Task Force Consensus Group. The metabolic syndrome - a new worldwide definition. Lancet 366: 1059-1062.

29. Mitchell M, Armstrong DT, Robker RL, Norman RJ, 2005 Adipokines: implications for female fertility and obesity. Reproduction 130: 583-597.

30. Pasquali R, Vicennati V, Gambineri A, 2002 Adrenal and gonadal function in obesity. J Endocrinol Invest 25: 893-898.

31. Bray GA, 1997 Obesity and reproduction. Hum Reprod 12: Suppl 1: 26-32.

32. Blóher S, Mantzoros CS, 2007 Leptin in reproduction. Curr Opin Endocrinol Diabetes Obes 14: 458-464.

33. Gao Q, Horvath TL, 2008 Cross-talk between estrogen and leptin signaling in the hypothalamus. Am J Physiol Endocrinol Metab 294: E817-826.

34. Margetic S, Gazzola C, Pegg GG, Hill RA, 2002 Leptin: a review of its peripheral actions and interactions. Int $\mathrm{J}$ Obes Relat Metab Disord 26: 1407-1433.
35. Lado-Abeal J, Norman RL, 2002 Leptin and reproductive function in males. Semin Reprod Med 20: 145-151.

36. Tena-Sempere M, Barreiro ML, 2002 Leptin in male reproduction: the testis paradigm. Mol Cell Endocrinol 188: 9-13.

37. Fischer-Posovszky P, Wabitsch M, Hochberg Z, 2007 Endocrinology of adipose tissue - an update. Horm Metab Res 39: 314-321.

38. Nestler JE, 2000 Obesity, insulin, sex steroids and ovulation. Int J Obes Relat Metab Disord 24: Suppl 2: 7173.

39. Haffner SM, 2000 Sex hormones, obesity, fat distribution, type 2 diabetes and insulin resistance: epidemiological and clinical correlation. Int J Obes Relat Metab Disord 24: Suppl 2: 56-58.

40. Tchernof A, Després JP, 2000 Sex steroid hormones, sex hormone-binding globulin, and obesity in men and women. Horm Metab Res 32: 526-536.

41. Norman RJ, Clark AM, 1998 Obesity and reproductive disorders: a review. Reprod Fertil Dev 10: 55-63.

42. Kokkoris P, Pi-Sunyer FX. 2003 Obesity and endocrine disease. Endocrinol Metab Clin North Am 32: 895914.

43. Balen AH, Anderson RA, 2007 Policy \& Practice Committee of the BFS. Impact of obesity on female reproductive health: British Fertility Society, Policy and Practice Guidelines. Hum Fertil (Camb) 10: 195-206.

44. Pasquali R, Patton L, Gambineri A, 2007 Obesity and infertility. Curr Opin Endocrinol Diabetes Obes 14: 482487.

45. Metwally M, Li TC, Ledger WL, 2007 The impact of obesity on female reproductive function. Obes Rev 8: 515-523.

46. Nelson SM, Fleming R, 2007 Obesity and reproduction: impact and interventions. Curr Opin Obstet Gynecol 19: 384-389.

47. Goulis DG, Tarlatzis BC, 2008 Metabolic syndrome and reproduction: I. testicular function. Gynecol Endocrinol 24: 33-39.

48. Yudkin JS, 2007 Inflammation, obesity, and the metabolic syndrome. Horm Metab Res 39: 707-709.

49. Hotamisligil GS, 2006 Inflammation and metabolic disorders. Nature 444: 860-867.

50. Tsigos C, Kyrou I, Chala E, et al, 1999 Circulating tumor necrosis factor alpha concentrations are higher in abdominal versus peripheral obesity. Metabolism 48: 1332-1335.

51. Trayhurn P, Wood IS, 2004 Adipokines: inflammation and the pleiotropic role of white adipose tissue. Br J Nutr 92: 347-355.

52. Hotamisligil GS, Spiegelman BM, 1994 Tumor necrosis factor alpha: a key component of the obesity-diabetes link. Diabetes 43: 1271-1278.

53. Tsigos C, Papanicolaou DA, Kyrou I, Defensor R, Mitsiadis CS, Chrousos GP, 1997 Dose-dependent effects of recombinant human interleukin-6 on glucose regulation. J Clin Endocrinol Metab 82: 4167-4170. 